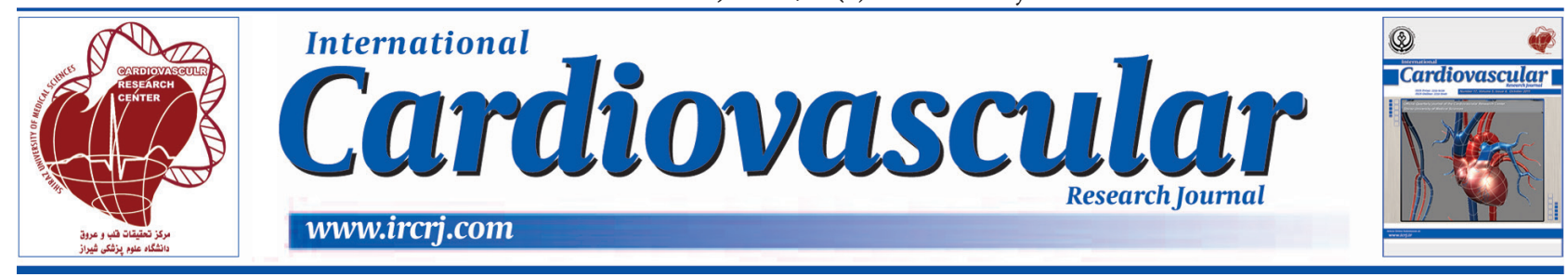

\title{
Ectatic-Fistulous Coronary Artery Lesion: A Newly Proposed Nomenclature
}

\author{
Ilias A. Kouerinis ${ }^{1, *}$, Mathew Benjamin ${ }^{1}$, Ioannis Karathanasis ${ }^{2}$, George Peskesis ${ }^{2}$, Charilaos Kantsos ${ }^{2}$, \\ Georgia Sarri ${ }^{2}$, Grigorios Karagkiouzis ${ }^{2}$, Uday Trivedi ${ }^{1}$
}

\footnotetext{
${ }^{1}$ Department of Cardiac Surgery, Brighton and Sussex University Hospitals, Brighton, UK

${ }^{2}$ Department of Cardiac Surgery, Hippokration Hospital, Athens, Greece
}

\begin{tabular}{|c|c|}
\hline A R T I C LE I N F O & A B S TRACT \\
\hline Article Type: & \multirow{10}{*}{$\begin{array}{l}\text { Congenital Coronary Artery Fistulae (CAF) originating from Right Coronary Artery } \\
\text { (RCA) and draining into Coronary Sinus (CS) demonstrate high gradients and high flows, } \\
\text { which result in ectasia of the entire artery. The anatomic margin between the ectatic RCA } \\
\text { and the fistula are not clear and most cardiologists and cardiac surgeons tend to misname } \\
\text { the entire vessel as "fistula". We presented a } 60 \text {-year-old female with multiple CAF } \\
\text { draining into CS. The fistula originating from the RCA was huge and caused severe ectasia } \\
\text { of the entire artery. The patient had progressive breathlessness due to high left to right } \\
\text { shunt. Both fistulae were ligated under cardiopulmonary bypass. Our introduced term } \\
\text { "ectatic-fistulous lesion" comes to address the confusion with nomenclature. It not only } \\
\text { offers the theoretical advantage of precise description of the above complex pathology, but } \\
\text { also allows the cardiac team to identify the anatomical margins between the ectasia and } \\
\text { the fistula and, thus, to proceed to closure of the coronary fistula with safety. }\end{array}$} \\
\hline Case Report & \\
\hline & \\
\hline Received: 01 Jan 2015 & \\
\hline Revised: 14 Mar 2015 & \\
\hline Accepted: 06 Apr 2015 & \\
\hline Keywords: & \\
\hline Fistula & \\
\hline $\begin{array}{l}\text { Surgery } \\
\text { Nomenclature }\end{array}$ & \\
\hline & \\
\hline
\end{tabular}

- Implication for health policy/practice/research/medical education:

Our introduced term, mixed ectatic-fistulous lesion, comes to address the confusion with nomenclature. It not only offers the theoretical advantage of precise description of the above complex pathology, but also allows the cardiac team to identify the anatomic margins between the ectasia and the fistula and, thus, to proceed to closure of the coronary fistula with safety.

\section{Introduction}

Coronary artery anomalies include coronary aneurysms, coronary artery ectasia, and coronary artery fistulae. In controversy to coronary artery aneurysms, which involve only part of the vessel, coronary artery ectasia (CE) is defined as an abnormal increase of $>1.5$ in the arterial diameter involving the entire artery. Additionally, Coronary Artery Fistula (CAF) refers to abnormal communication of a coronary arterial branch with a ventricular chamber or a major vessel (1).

Congenital CAF originating from Right Coronary Artery (RCA) and draining into Coronary Sinus (CS) shows high gradient and high flow within the native artery, which then becomes ectatic. Determination of the anatomic margins between the coronary ectasia and the fistula has not been studied yet and closure of such a

*Corresponding author: Ilias A. Kouerinis, Sussex House, 37 St George Road, BN2 1ED, Brighton, UK, Tel: 0030-6932713171, Fax: 0030-2132088595,

E-mail: ikouerinis@hotmail.com fistula has remained empirical.

\section{Case Presentation}

A 60-year-old female was admitted to our department with diagnosis of two CAF and left anterior descending stenosis. One of the fistulae was huge and originated from an ectatic RCA (Figure 1), while the other one was much smaller and originated from an obtuse marginal branch. Both CAF were draining into the CS. The patient had progressive breathlessness (NYHA II to III), which was attributed to the giant fistula, as the Left Anterior Descending (LAD) artery stenosis, and the smaller fistula was considered to be of lesser clinical importance.

At operation, the serpentine motion of the ectatic RCA (Figure 2) was evident under the intact pericardium. After opening the pericardium and lifting the heart, we identified the termination point of the fistulae into CS. None of the CAF was calcified. This facilitated our preoperative plan for ligation of the main fistula on a beating heart in order 


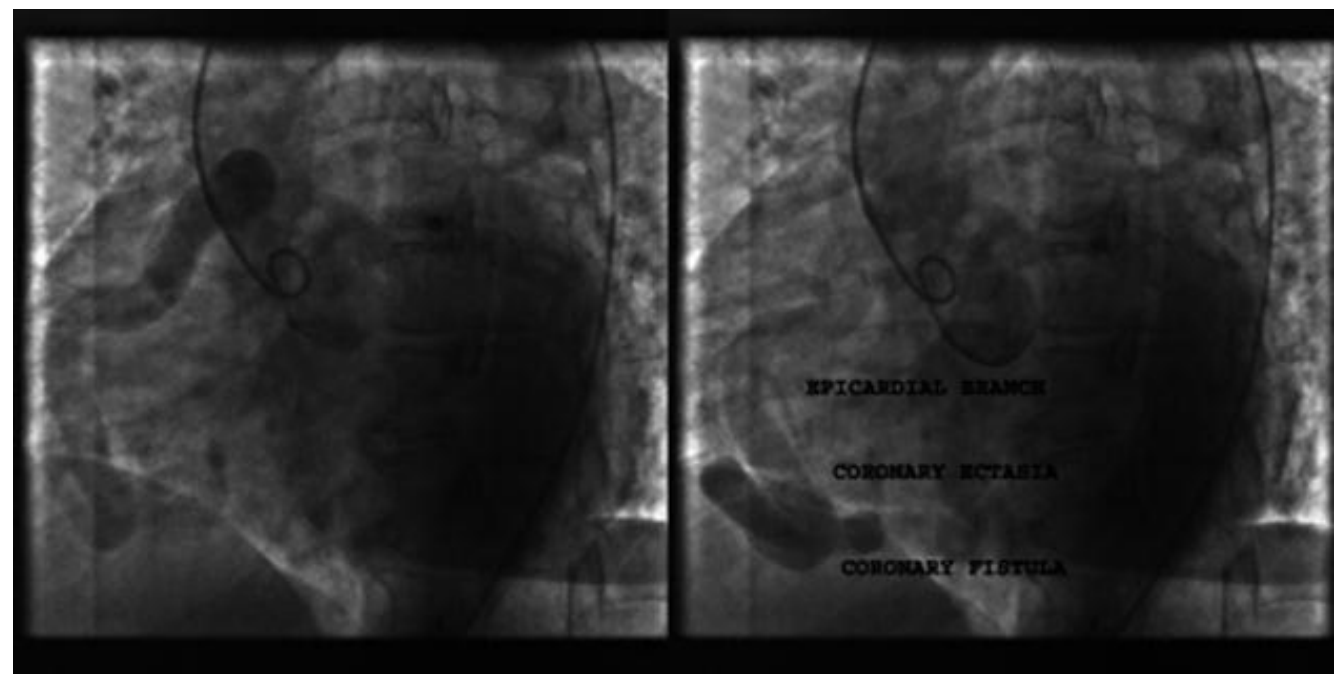

Figure 1. The Coronary Angiogram Showing the Ectatic RCA and the Fistula Draining into the Coronary Sinus

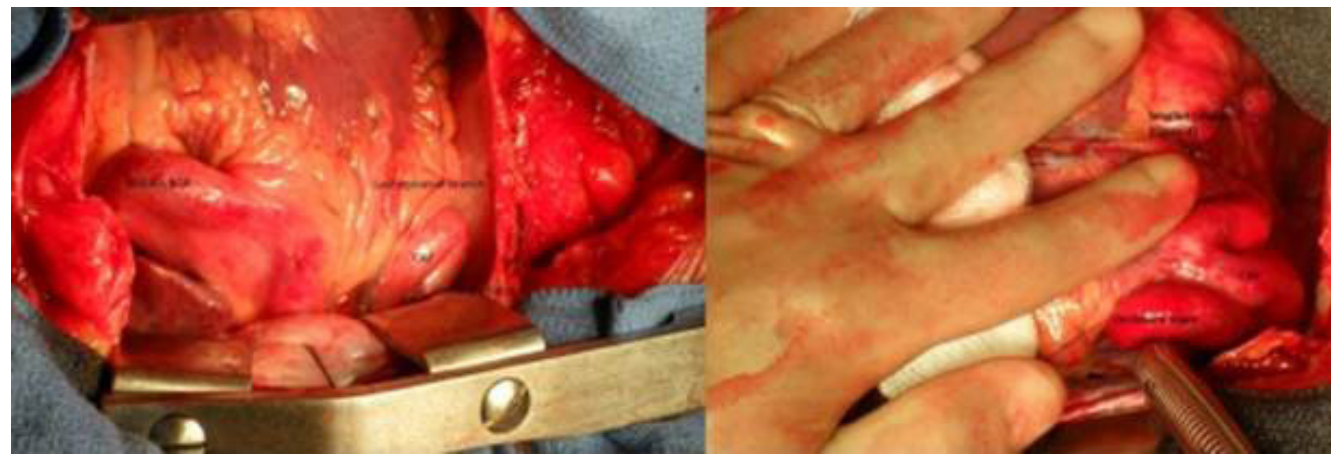

Figure 2. a) Our Intraoperative Findings. The Anatomical Margin between the Ectatic RCA and the Fistula Is Indicated from the Presence of the Last Epicardial Branch, Which Was an Acute Marginal one in our Patient. b) The Smaller Fistulae Were Ligated under Cardiopulmonary Bypass. A Snare Was Positioned around the Fistula, Which Was Going to be Ligated in a Beating State

to detect any possible myocardial ischemia. The shunt $(\mathrm{Qp} /$ Qs) was measured at 1.4 and was calculated by taking blood samples from the aorta, superior vena cava, pulmonary vein, and pulmonary artery according to Fick formula: $\mathrm{Qp} / \mathrm{Qs}=($ SatAorta- SatSVC)/(SatPulmonary Venous SatPulmonary Artery). The left internal mammary artery was harvested and anastomosed to the LAD under cardiac arrest. Subsequently, the smaller fistula was ligated close to the CS. The cross clamp was removed, Sinus Rhythm (SR) was re-established, and the giant CAF was snared with a tourniquet distal to the origin of the last epicardial branch, which was the acute marginal branch (Figure 3). No ischemia was noticed on the Electrocardiogram (ECG) and the CAF was ligated with multiple Prolene 4-0 sutures. Repeated measurement of Qp/Qs was done at 1.02, which was acceptable. The patient had an uneventful postoperative period and was discharged in excellent conditions.

\section{Discussion}

Apart from their impressive anatomy, CAF have an even more interesting pathophysiology. According to our previously proposed classification (2), CAF with $\mathrm{CS}$ as the termination point are considered to be extramyocardial. These fistulae show left to right shunt with a continuous murmur that is more intense during systole. Larger CAF are susceptible to right heart failure due to volume overloading of the right ventricle, but their ischemic impact is less important as the major shunt occurs during systole (the reverse steal phenomenon occurs during diastole) (3). These high gradient-high flow CAF do not tend to close spontaneously. Coronary ectasia is the usual hemodynamic result of such fistulae originating from the RCA and draining into the CS $(4,5)$. The primary fistulous congenital anomaly alternates the hemodynamics within the RCA and the chronic presence of high flows within the vessel makes it ectatic. The more ectatic the artery becomes with progress of time, the more severe the symptoms from the increased left to right shunt will be (2).

Ectatic RCA is at the continuation of the fistula and their anatomic margins are not clearly determined. Such a determination is beyond theory as the surgeon and the interventionist have to identify where the artery ends in order to decide where to occlude the fistula. This became obvious in all the presented cases where the coronary artery lesion looked but was not uniform. According to our proposal, the coronary ectasia ends where the last epicardial arterial branch can be seen from the surgeon or can be obscured by the interventionist and/or where the vessel deviates from its predicted course in order to drain into the CS. This is reasonable as ectatic coronaries, although diseased, do participate in myocardial perfusion, while CAF are true shunts and do not. It is also interesting that the arterial branches that originate from the ectatic RCA are all of normal dimensions. 
After identification of the margin between the ectasia and the fistula, it is always advisable to temporarily occlude the CAF in order to detect any ischemia. The surgeon may achieve it with a snare and the interventionist with an occlusive balloon. Aggressive attempts to close the fistula as close to the drain site as possible may result in occlusion of the CS and, consequently, it is considered to be of increased risk.

In conclusion, the term "ectatic-fistulous lesion" describes the secondary dilatation of RCA due to a high gradienthigh flow congenital fistula, which drains into the CS. In this regard, identification of the last anatomic branch is of paramount importance as it shows the margin between the above lesions and indicates a safe point for closure of the abnormal communication.

\section{Acknowledgements}

There is no acknowledgement.

\section{Authors' Contribution}

Ilias Kouerinis, Mathew Benjamin, Ioannis Karathanassis, and Uday Trivedi: study concept and design; Charilaos Kantsos, Grigorios Karagkiouzis, and George Peskesis: Drafting of the manuscript; Georgia Sarri and Uday Trivedi:
Study supervision

\section{Funding/Support}

There is no support.

\section{Financial Disclosure}

There is no financial disclosure.

\section{References}

1. Mavrogeni S. Coronary artery ectasia: from diagnosis to treatment. Hellenic J Cardiol. 2010;51(2):158-63.

2. Kouerinis IA, Deligianni M, Maniatis V, Elefteriadis I, Kourtesis A, Michalopoulos N. Giant congenital left main coronary artery fistula to the superior vena cava in an adult patient with coronary artery disease. J Thorac Cardiovasc Surg. 2007;134(4):1057-8.

3. Bamoshmoosh M, Marraccini P, Pratali L, Ciriello G, Ciardetti M, Mazzarisi A. "Reverse steal phenomenon" in a patient with coronary artery disease and coronary-left ventricular fistula. Int $J$ Cardiol. 2007;115(1):e33-5.

4. Yu L, Gu T, Shi E, Xiu Z, Fang Q, Liu B. Surgical repair of coronary artery fistula combined with coronary artery ectasia in adults. $J$ Card Surg. 2013;28(3):222-7.

5. Yu L, Shi E, Gu T. Aneurysmal right coronary with fistula to the coronary sinus combined with severe stenosis of the left anterior descending artery: a snake on the heart. J Thorac Cardiovasc Surg. 2011;142(4):937-9. 